OPEN ACCESS

Edited by:

Tao Liu,

Children's Cancer Institute Australia,

Australia

Reviewed by:

Silvia Carolina Galvan, National Autonomous University

of Mexico, Mexico

Jessica Lilian Bell,

Martin Luther University of

Halle-Wittenberg, Germany

${ }^{*}$ Correspondence:

Guang Yang

tyangguang@email.jnu.edu.cn

Chunxia Jing

jcxphd@gmail.com

tThese authors have contributed equally to this work

Specialty section: This article was submitted to Epigenomics and Epigenetics,

a section of the journal

Frontiers in Genetics

Received: 22 December 2018 Accepted: 16 October 2019 Published: 14 November 2019

Citation:

Huang S, Li R, Huang $X$, Zheng $S$,

Wang L, Wen Z, Zou X, Wu J, Liu Y,

Liu D, Wang $Y$, Dong S, Chen $X$,

Zhu K, Du X, Zhou Z, Han Y, YeX,

Zeng $C$, Zhang $B$, Yang $G$ and Jing $C$

(2019) Association Study Between

Methylation in the Promoter Regions

of CGAS, MAVS, and TRAF3

Genes and the Risk of Cervical

Precancerous Lesions and

Cervical Cancer in a Southern

Chinese Population.

Front. Genet. 10:1123.

doi: 10.3389/fgene.2019.01123

\section{Association Study Between Methylation in the Promoter Regions of cGAS, MAVS, and TRAF3 Genes and the Risk of Cervical Precancerous Lesions and Cervical Cancer in a Southern Chinese Population}

Shiqi Huang ${ }^{1 \dagger}$, Ruixin $\mathrm{Li}^{2 \dagger}$, Xiuxia Huang ${ }^{1 \dagger}$, Shaoling Zheng ${ }^{1 \dagger}$, Lijun Wang ${ }^{3}$, Zihao Wen ${ }^{1}$, Xiaoqian Zou', Jing Wu' ${ }^{1}$, Yumei Liu' ${ }^{1}$, Dandan Liu' ${ }^{1}$, Yao Wang ${ }^{1}$, Shirui Dong ${ }^{1}$, Xiaojing Chen ${ }^{1}$, Kehui Zhu' ${ }^{1}$ Xiuben Du' ${ }^{1}$, Zixing Zhou' ${ }^{1}$, Yajing Han' ${ }^{1}$, Xiaohong Ye ${ }^{1}$, Chengli Zeng ${ }^{1}$, Baohuan Zhang ${ }^{1}$, Guang Yang ${ }^{4,5 *}$ and Chunxia Jing ${ }^{1,5 *}$

${ }^{1}$ Department of Epidemiology, School of Medicine, Jinan University, Guangzhou, China, ${ }^{2}$ Department of Gynecologic Oncology, Sun Yat-sen Memorial Hospital, Sun Yat-sen University, Guangzhou, China, ${ }^{3}$ Department of Nutriology, School of Medicine, Jinan University, Guangzhou, China, ${ }^{4}$ Department of Pathogen Biology, School of Medicine, Jinan University, Guangzhou, China, ${ }^{5}$ Guangdong Key Laboratory of Environmental Pollution and Health, Jinan University, Guangzhou, China

A case-control study was used to explore the association between the methylation status in the promoter regions of the cGAS, MAVS, and TRAF3 genes and the diseases of cervical precancerous lesions (CPL) and cervical cancer (CC) in a Southern Chinese population, and to further explore their interaction effects with high-risk human papillomavirus (hrHPV) infection and environmental factors in these diseases. The study protocol was approved by the ethics committee of The First Affiliated Hospital of Jinan University, and this study was performed in 97 healthy controls, 75 patients with CPL and 33 patients with CC, while each participant has read and signed the informed consent forms before enrolment. The promoter methylation status genes were detected from the bisulfite-treated DNA by the bisulfite sequencing PCR (BSP) technique, which was carried out using MethPrimer. The cGAS, MAVS, and TRAF3 promoter methylation levels in $C P L\left(C P L_{c G A S}=35.40 \%\right.$, $\mathrm{CPL}_{\text {MAVS }}=24.26 \%$, and $\mathrm{CPL}_{\text {TRAF3 }}=96.76 \%$ ) were significantly higher than those in the control (Control ${ }_{C G A S}=31.87 \%$, Control ${ }_{\text {MAVS }}=21.16 \%$, and Control ${ }_{\text {TRAF3 }}=96.26 \%, P_{C G A S}<$ $0.001, P_{\text {MAVS }}<0.001$, and $P_{\text {TRAF3 }}=0.001$ ); however, there was no significant differences between the CC and control. In the logistic regression model with adjusted covariates, compared with the individuals whose cGAS methylation levels were less than or equal to $31.87 \%$, the women with the levels more than $31.87 \%$ increased the risk of CPL by 2.49 times $\left(\mathrm{OR}^{\mathrm{a}}=2.49,95 \% \mathrm{Cl}=1.31-4.75, \mathrm{~Pa}^{\mathrm{a}}=0.006\right)$. The women with MAVS methylation levels above $21.16 \%$ were 1.97 times more likely to have $\mathrm{CPL}$ than the those with the levels less than $21.16 \%\left(\mathrm{OR}^{\mathrm{a}}=1.97,95 \% \mathrm{Cl}=1.06-3.69, \mathrm{P}^{\mathrm{a}}=0.033\right)$. A synergistic interaction was found between hrHPV and gene promoter methylation levels of $c G A S$ 
and MAVS in CPL; however, no potential interaction was observed in CC. The promoter methylation levels in CGAS, MAVS, and TRAF3 genes are higher in CPL than in control, indicating that hypermethylation might be an early event in the progression of cervical intraepithelial neoplasia (CIN). The interaction between the promoter methylation levels in cGAS and MAVS genes and hrHPV infection might play a role in the development of CPL.

Keywords: cervical precancerous lesions, cervical cancer, cGAS, MAVS, TRAF3, gene promoter methylation, interaction

\section{INTRODUCTION}

Abnormal DNA methylation in the gene promoter region is a well-recognized epigenetic hallmark in the premalignant and malignant stages of cancers, and it has been observed in different kinds of genes, such as tumor suppressor genes and DNA repair genes (Merlo et al., 1995; Esteller et al., 1999a; Esteller et al., 2000; Guo et al., 2009; Zou et al., 2009; Esteller et al., 1999b). Methylation of the CpG sites in promoter regions can lead to gene dysfunction or inactivation, causing tumor progression (Nojima et al., 2001; Ribeiro-Filho et al., 2002). Several studies have reported that promoter methylation status of multiple genes is associated with cervical precancerous lesions (CPL) and cervical cancer (CC) development, such as CDH13, CDKN2B, TIM3, and RASSF1A (Dong et al., 2001; Missaoui et al., 2011a; Li et al., 2015a). Methylation has been detected at cervical precancerous stages, and methylated DNA is a relatively stable target and allows for flexibility of assay development, suggesting that methylation markers may have value in cervical diseases screening (Wentzensen et al., 2009; Steenbergen et al., 2014). DNA methylation of promoter regions is associated with the cervical disease, but very few studies have been reported for gene promoter methylation related to antiviral innate immunity pathways, moreover, the research evaluating DNA methylation and epidemiologic factors for CPL and CC is very limited in Chinese women.

$\mathrm{CC}$ is one of the most common malignancies in women, with an estimated 530,000 new cases and 275,000 deaths worldwide per year (Zhao et al., 2012). Approximately $90 \%$ of the deaths from CC occurred in low- and middle-income countries during 2015. The mortality of CC has significantly decreased in China, but a younger age trend and a substantial increase in CC incidence have been seen in some regions (Shi et al., 2012; Chen et al., 2016). The incidence and mortality of cervical disease vary widely among different populations, geographic areas and time periods in China (Li et al., 2011). The incidence and mortality rates of CC could be reduced through effective screening, early prevention and diagnosis (Peto et al., 2004b). High-risk human papillomavirus (hrHPV) is the cause of CPL and CC (Mj et al., 2005), and hrHPV prevalence is well correlated with the risk of diseases, particularly in middle-age women (Peto et al., 2004a; Maucort-Boulch et al., 2008; Bouvard et al., 2009). The squamous intraepithelial lesions (SILs) of the cervix are referred to as CPL, which can be divided into low-grade SIL (LSIL or cervical intraepithelial neoplasia 1, CIN1) and highgrade SIL (HSIL or CIN2/3) histologically. The progression from LSIL through HSIL and eventually to invasive cancer lasts up to
10-30 years (Winer et al., 2005; Lendvai et al., 2012). The periods of CPL are generally chronic and reversible; therefore, various methods can be adopted to prevent carcinogenesis, and the stage of CPL might be affected by many genetic or environmental factors (Armstrong and Doll, 1975).

Viral infection of the host can trigger innate immune responses, and previous studies have revealed that cyclic GMPAMP synthase (cGAS)/STING and RIG-I/MAVS pathways play important roles in host innate immunity against $\mathrm{HPV}$-induced precursor lesions and invasive cancer of the uterine cervix (Karim et al., 2013; Lau et al., 2015; Xiao et al., 2016). cGAS, also known as MB21D1 or C6orf150, is identified as a general cytosolic DNA sensor, which can detect intracellular DNA and catalyze the synthesis of second messenger cyclic-GMP-AMP (cGAMP) from ATP and GTP (Sun et al., 2013). Then synthesized cGAMP binds to and activates the adaptor protein STING (stimulator of interferon genes) to induce the phosphorylation of downstream factors, including TANK binding kinase 1 (TBK1) and interferon regulatory factor 3 (IRF3), thereby triggering type-I interferon (IFN) production (Wu et al., 2013; Lau et al., 2015). Type I IFNs are critical for antiviral autoimmune responses. The RIG-I/ $M A V S$ pathway can also mediate IFN production in response to cytosolic double-stranded RNA or single-stranded RNA containing 5'-triphosphate (5'-ppp) (Kawai et al., 2005; Hornung et al., 2006). RNA polymerase III (Pol-III) has been suggested to function as a potential DNA sensor that can identify and convert cytosolic DNA into 5'-triphosphate (5'-ppp) RNA (Hornung et al., 2006; Pichlmair et al., 2006; Chiu et al., 2009), which can be detected by RIG-I sensor and lead to the mitochondrial assembly of mitochondrial antiviral-signaling (MAVS) complexes. Then, $M A V S$ binds to tumor necrosis factor receptor-associated factor 3 (TRAF3) and recruits TBK1/IKKe kinases that activate NF- $\kappa B$ and direct the phosphorylation of constitutively expressed IRF3 to induce IFN- $\beta$ (Fitzgerald et al., 2003; Oganesyan et al., 2006; Hiscott, 2007; Matthys et al., 2014).

In this study, we investigated the promoter methylation status of $c G A S, M A V S$, and TRAF3 in CPL and CC, and we further explored the association between potential biological interactions and the risk of CPL and CC in the Southern Chinese population.

\section{MATERIALS AND METHODS}

\section{Study Participants}

A total of 205 Southern Chinese women were recruited in our study, including 97 healthy women in the control group and 
108 patients with CPL and CC. The mean age was 45.50 years, ranging from 20 to 65 years. The classifications of the groups were according to the ThinPrep cytologic test (TCT) with the Bethesda system for reporting cervical cytology (TBS 2001) and cervical biopsies were confirmed by two pathologists. The women in the control group were negative for CPL or CC without other diseases of uterus or cervix. The CPL group $(n=75)$ was women with SILs, including 38 with LSILs and 37 with HSILs. There were 33 patients in the CC group.

Epidemiological data were obtained from the face-to-face questionnaire of each study subject; these questionnaires were conducted by qualified investigators and a doctor to ensure the accuracy and authenticity of the information. In addition, peripheral venous blood and cervical epithelial exfoliated cells were also collected from each participant. The blood sample was gathered with EDTA vacuum collection tubes and maintained at 4 degrees; then, blood samples were transported to our laboratory for DNA and RNA extraction. Cervical epithelial exfoliated cells were collected by cytobrush (QIAGEN, Valencia, CA) and preserved in denaturation buffer. ThinPrep 2000 (Hologic Inc.) and SurePath liquidbased Pap test (BD, USA) were used for the TCT. HPV DNA from cervical samples was extracted from the commercial magnetic beads kit (Chemagen, PerkinElmer, Waltham, MA), which was performed in the clinical standard laboratory of BGI (Beijing Genomics Institute, Shenzhen, China). Both hrHPV (types 16, 18, 31, 33, 35, 39, 45, 51, 52, 56, 58, 59, 66, and 68) and low-risk HPV (lrHPV, types 6 and 11) were detected with MassARRAY (Sequenom, Sandiego, CA) based on the matrix-assisted laser desorption/ionization time of flight (MALDI-TOF) mass spectrometry (MS).

\section{Methylation Status Detection}

The promoter region is approximately 2000-bp length upstream of the gene transcription start site, and the reference promoter sequences for the human $c G A S, M A V S$, and TRAF3 genes are obtained from the UCSC Gene Sorter (http://www. genome.ucsc.edu/cgi-bin/hgNear). The methylation status is described as percentage of methylated CpGs of the possible CpG methylation sites, which is detected by the bisulfite sequencing PCR (BSP) technique. The BSP is the most widely used method to give information about the methylation profile of every single $\mathrm{CpG}$ site in a given sequence. We use MethPrimer (http://www.urogene.org/methprimer/indexl. html) to carry out the bisulfite transformation of the target sequences and design primers (Li and Dahiya, 2002), which is based on key CpG island prediction in the promoter regions of genes $c G A S / M A V S / T R A F 3$ (Figure 1).

Blood DNA was extracted using the QIAamp DNA Mini Kit (QIAGEN, 51104), and the bisulfite-treated DNA was purified using the Wizard DNA Clean-up System (Promega, A7290) after DNA denaturation and bisulfite conversion. The DNA was PCRamplified by using the EpiTaq HS Kit (TaKaRa, R110A) in a 20- $\mu$ l reaction volume with the designed primers (Table S1). The PCR conditions consisted of an initial incubation for $3 \mathrm{~min}$ at $98^{\circ} \mathrm{C}$, followed by $40 \mathrm{cycles}$ of $10 \mathrm{~s}$ at $98^{\circ} \mathrm{C}, 30 \mathrm{~s}$ at $55^{\circ} \mathrm{C}$, and $30 \mathrm{~s}$ at $72^{\circ} \mathrm{C}$, and finally $10 \mathrm{~min}$ at $74^{\circ} \mathrm{C}$. The PCR product was sequenced by IGE (Guangzhou IGE Biotechnology, LTD., China), and the sequencing results were analyzed using Chromas software. There were 9,10 , and $5 \mathrm{CpG}$ sites detected in the promoter region of the cGAS, MAVS, and TRAF3 genes, respectively. The methylation status was dependent on the peak height of cytosine (C) and thymine $(\mathrm{T})$ signals according to the following formula:

The methylation rate of single CpG site (\%)

$$
=\frac{\text { Peak height of } \mathrm{C}}{\text { Peak height of } \mathrm{C}+\text { Peak height of } \mathrm{T}} \times 100 \%
$$

The methylation level $(\%)=\frac{\text { Methylation rate of total CpG sites }}{\text { Number of CpG sites }}$

\section{Statistical Analyses}

The demographic and experimental data were obtained and recorded, and the database was established by Epidata software. Data analyses were performed with SPSS software (version 24.0, Inc., Chicago, USA). Differences among the groups of control, CPL, and CC were assessed by analysis of variance (ANOVA), Dunnett's t-test, chi-squared test, and Mann-Whitney U test. Correlation analysis was conducted using the logistic regression model, which was calculated by estimating the odds ratios (ORs) and 95\% confidence intervals (95\% CIs) after adjusting for potential covariates. Potential two-factor interaction was evaluated on an additive scale with a $95 \%$ CI by calculating the following three measures: synergy index (S), attributable proportion due to interaction (AP), and relative excess risk due to interaction (RERI) (Skrondal, 2003; Andersson et al., 2005). If there were no biological interactions, then the 95\% CI of S is over 1, and RERI and AP are cross 0 (Assmann et al., 1996; Andersson et al., 2005). Multifactor dimensionality reduction software 1.0.0 (MDR 1.0.0) was used to explore the multifactor interactions, and the best model was determined by the testing balanced accuracy (TBA) and cross-validation consistency (CVC) indexes. All $p$ values below 0.05 were considered statistically significant.

\section{RESULT}

\section{Demographic Characteristics and Relevant Factors Analysis}

Demographic characteristics and analysis of relevant factors are shown in Table 1. The patients in the CC group were older than in the control group, and the number of pregnancies was higher in the CC group than in the control group $(p<0.05)$. The positive rates of hrHPV in both the CPL (74.67\%) and CC $(82.35 \%)$ groups were significantly higher than in the control group (53.61\%). However, there were no significant differences in other factors among the groups $(P>0.05)$. 

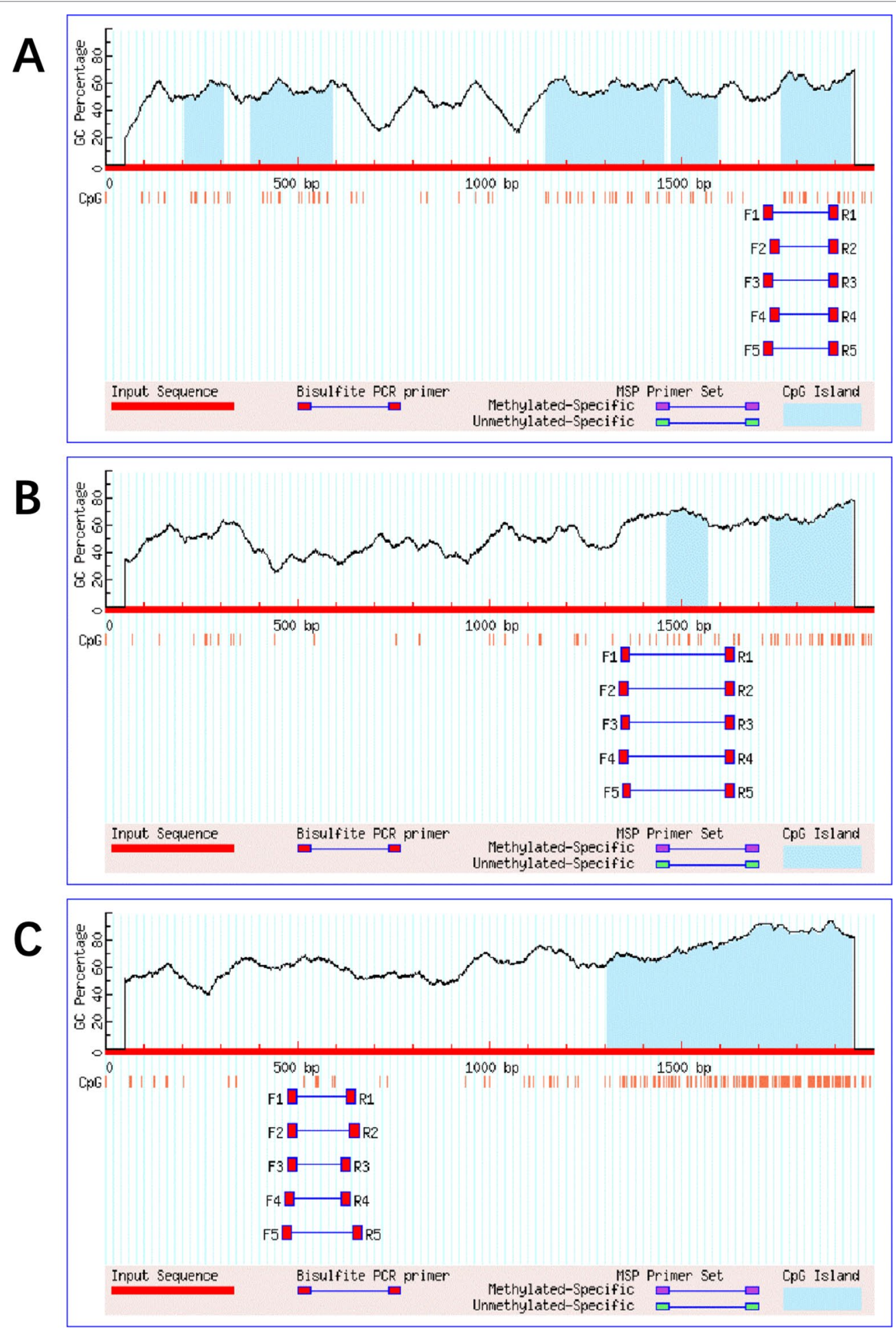

FIGURE 1 | The target sequences' bisulfite transformation and design primers in the promoter regions of genes cGAS/MAVS/TRAF3.

\section{Gene Promoter Methylation Status in Control, CPL, and CC}

The methylation rate of a single CpG site and the methylation levels of gene promoter regions are presented in Table 2. The methylation status of the single $\mathrm{CpG}$ site was as follows: the methylation level of the $c G A S, M A V S$, and TRAF3 genes and the methylation rates of the C1-C8 CpG sites of $c$ GAS, M1-M9 CpG sites of MAVS, and T1-T4 CpG sites of TRAF3 were higher in the CPL group than in the control group $(P<0.05)$; $\mathrm{C} 1$ and $\mathrm{C} 2$ in $c G A S, \mathrm{M} 4$ and M10 in MAVS, as well as T3 in TRAF3 were 
TABLE 1 | Demographic characteristics in control, CPL, and CC.

\begin{tabular}{|c|c|c|c|c|c|}
\hline Variables & Control $\mathbf{N}=97$ & CPL N = 75 & $C C N=33$ & $\mathbf{F} / \chi^{2}$ & $p$ value \\
\hline Age & $44.24 \pm 7.14^{b}$ & $44.52 \pm 7.35^{c}$ & $51.61 \pm 9.88^{b}$ & 12.212 & $<0.001$ \\
\hline $\mathrm{BMl}$ & $22.02 \pm 3.31$ & $22.06 \pm 2.55$ & $22.43 \pm 3.05$ & 0.241 & 0.786 \\
\hline Age at menarche & $15.21 \pm 1.74$ & $15.47 \pm 2.64$ & $14.32 \pm 1.54$ & 3.047 & 0.050 \\
\hline Age at first intercourse & $22.47 \pm 3.97$ & $22.63 \pm 3.18$ & $22.63 \pm 2.91$ & 0.048 & 0.953 \\
\hline Age at primiparity & $23.81 \pm 3.40$ & $23.88 \pm 3.58$ & $23.06 \pm 5.36$ & 0.551 & 0.577 \\
\hline Number of pregnancies & $3.02 \pm 1.28^{\mathrm{b}}$ & $3.25 \pm 1.39^{\circ}$ & $3.72 \pm 1.63^{b}$ & 3.141 & 0.045 \\
\hline Number of births & $2.38 \pm 0.93$ & $2.67 \pm 1.78$ & $2.69 \pm 1.47$ & 1.725 & 0.181 \\
\hline Number of abortions & $0.64 \pm 0.88$ & $0.59 \pm 0.95$ & $1.03 \pm 1.36$ & 2.423 & 0.091 \\
\hline \multicolumn{6}{|l|}{ HrHPV infection } \\
\hline Negative & 45 (46.39) & 19 (25.33) & $3(17.65)$ & 10.784 & 0.005 \\
\hline Positive & $52(53.61)^{\mathrm{b}}$ & $56(74.67)^{\mathrm{b}}$ & $14(82.35)^{b}$ & & \\
\hline Unavailable & 0 & 0 & 16 & & \\
\hline \multicolumn{6}{|l|}{ Passive smoking } \\
\hline Negative & 38 (39.20) & $24(32.00)$ & $12(41.40)$ & 1.24 & 0.538 \\
\hline Positive & $59(60.80)$ & $51(68.00)$ & $17(58.60)$ & & \\
\hline Unavailable & 0 & 0 & 4 & & \\
\hline \multicolumn{6}{|l|}{ Physical exercise } \\
\hline Negative & $84(86.60)$ & 67 (89.30) & $25(75.76)$ & 3.562 & 0.169 \\
\hline Positive & $13(13.40)$ & $8(10.70)$ & $8(24.24)$ & & \\
\hline
\end{tabular}

Data is shown means \pm standard deviation for continuous variable, and the number of samples is shown for categorical variable. $P$ value is based on ANOVA or Chisquare test. Bold values indicate the statistically significant $p$ value $(p<0.05)$. The same letter of b/c indicates that the significant differences compared with the control group. CPL, cervical precancerous lesions; CC, cervical cancer; HrHPV, high-risk HPV; BMl, body mass index, $\mathrm{kg} / \mathrm{m}^{2}$. HrHPV positive is including HPV negative and low-risk HPV positive.

TABLE 2 | Promoter methylation status of cyclic GMP-AMP synthase (cGAS)/mitochondrial antiviral-signaling (MAVS)/tumor necrosis factor receptor-associated factor 3 (TRAF3) in control, cervical precancerous lesion (CPL), and cervical cancer (CC).

\begin{tabular}{|c|c|c|c|c|c|}
\hline \multirow[t]{2}{*}{ Methylation status } & \multicolumn{3}{|c|}{ Median (\%) } & \multicolumn{2}{|c|}{$p$ value } \\
\hline & Control & CPL & CC & Control/CPL & Control/CC \\
\hline \multicolumn{6}{|l|}{ CpG site of cGAS } \\
\hline C1 & 62.09 & 66.29 & 67.38 & 0.001 & 0.003 \\
\hline $\mathrm{C} 2$ & 50.14 & 54.24 & 53.95 & 0.005 & 0.012 \\
\hline C3 & 24.37 & 30.50 & 19.57 & 0.001 & 0.238 \\
\hline $\mathrm{C} 4$ & 25.51 & 28.00 & 23.47 & 0.036 & 0.837 \\
\hline C5 & 29.87 & 38.37 & 29.00 & $<0.001$ & 0.904 \\
\hline C6 & 31.98 & 40.17 & 31.58 & 0.002 & 0.523 \\
\hline $\mathrm{C} 7$ & 32.33 & 38.65 & 29.81 & $<0.001$ & 0.673 \\
\hline $\mathrm{C} 8$ & 19.11 & 26.24 & 17.29 & $<0.001$ & 0.554 \\
\hline $\mathrm{C9}$ & 8.57 & 9.00 & 6.18 & 0.477 & 0.056 \\
\hline Methylation level of $c G A S$ & 31.87 & 35.40 & 29.75 & $<0.001$ & 0.925 \\
\hline \multicolumn{6}{|l|}{ CpG site of MAVS } \\
\hline M1 & 12.46 & 15.46 & 12.67 & 0.005 & 0.808 \\
\hline M2 & 5.70 & 7.93 & 7.26 & 0.003 & 0.121 \\
\hline M3 & 8.41 & 10.34 & 10.79 & 0.019 & 0.165 \\
\hline M4 & 5.09 & 6.93 & 6.79 & 0.002 & 0.026 \\
\hline M5 & 23.87 & 28.07 & 24.69 & 0.003 & 0.415 \\
\hline M6 & 54.19 & 56.70 & 55.07 & 0.005 & 0.313 \\
\hline M7 & 51.73 & 53.62 & 53.01 & 0.005 & 0.242 \\
\hline M8 & 24.32 & 26.92 & 23.30 & 0.042 & 0.433 \\
\hline M9 & 12.25 & 16.12 & 14.24 & 0.007 & 0.462 \\
\hline M10 & 6.58 & 6.98 & 9.53 & 0.160 & 0.032 \\
\hline Methylation level of MAVS & 21.16 & 24.26 & 22.12 & $<0.001$ & 0.236 \\
\hline \multicolumn{6}{|l|}{ CpG site of TRAF3 } \\
\hline T1 & 95.48 & 95.96 & 95.71 & 0.013 & 0.606 \\
\hline T2 & 98.12 & 100.00 & 100.00 & 0.021 & 0.079 \\
\hline T3 & 97.21 & 100.00 & 100.00 & 0.006 & 0.006 \\
\hline T4 & 97.11 & 97.98 & 96.73 & 0.019 & 0.206 \\
\hline T5 & 93.03 & 93.65 & 92.76 & 0.227 & 0.047 \\
\hline Methylation level of TRAF3 & 96.26 & 96.76 & 96.65 & 0.001 & 0.324 \\
\hline
\end{tabular}

Statistically significant $p$ values $(p<0.05)$ in bold font were based on the Mann-Whitney $U$ test. 
higher in the CC group than in the control group $(P<0.05)$. The methylation levels of the $c G A S, M A V S$, and TRAF3 gene promoter regions in the CPL group $\left(\mathrm{CPL}_{c G A S}=35.40 \%, \mathrm{CPL}_{M A V S}=24.26 \%\right.$, and $\mathrm{CPL}_{\text {TRAF3 }}=96.76 \%$ ) were significantly higher than in the control group $\left(\right.$ Control $_{c G A S}=31.87 \%$, Control ${ }_{M A V S}=21.16 \%$, and Control $_{\text {TRAF3 }}=96.26 \% ; P_{c G A S}<0.001, P_{M A V S}<0.001$, and $P_{T R A F 3}=$ 0.001 ), while there were no significant differences between the CC and control groups (Figure 2).

\section{Association of the Gene Promoter Methylation Status With the Risk of CPL and CC}

We investigated the relationship between the gene promoter methylation status of $c G A S, M A V S$, and TRAF3 and the diseases of CPL and CC. The methylation levels in both $c G A S$ and MAVS were associated with CPL after adjusting for age and number of pregnancies, but no associations were observed between the methylation levels and CC group (Table 3). Compared with the individuals whose methylation levels in $c G A S$ were less than or equal to $31.87 \%$, the other individuals had an increased risk of CPL by 2.49 times $\left(\mathrm{OR}^{\mathrm{a}}=2.49,95 \% \mathrm{CI}=1.31-4.75, P^{\mathrm{a}}=0.006\right)$. Moreover, individuals with MAVS methylation levels above $21.16 \%$ had a 1.97 times higher risk of CPL than the others $\left(\mathrm{OR}^{\mathrm{a}}\right.$ $\left.=1.97,95 \% \mathrm{CI}=1.06-3.69, P^{\mathrm{a}}=0.033\right)$. After adjusting for age and number of pregnancies, C1, C2, C3, C5, C6, C7, and C8 of cGAS, M2, M3, M5, M6, M7, and M9 of MAVS and T2, T3, T4, and $\mathrm{T} 5$ of TRAF3 were associated with CPL, and $\mathrm{C} 1, \mathrm{C} 2, \mathrm{C} 8$, and C9 of $c G A S$ were associated with the CC group (Table S2).

\section{Association between the Biological Interaction and the Diseases of CPL and CC}

There were no relationships between hrHPV and gene promoter methylation levels in the different groups (Table S3); therefore, we further explored the biological interactions of hrHPV and methylation levels and the estimated risk of CPL and CC. A synergistic interaction was found between the presence of hrHPV and the gene promoter methylation levels in $c G A S$ as well as in MAVS in CPL $\left(\mathrm{AP}_{c G A S}^{\mathrm{a}}=0.54,95 \% \mathrm{CI}=0.13-0.95 ; \mathrm{AP}^{\mathrm{a}}{ }_{M A V S}=\right.$ $0.50,95 \% \mathrm{CI}=0.01-0.99)$, and no potential interactions were observed in CC (Table S4).

The interactions of the presence of hrHPV and methylation levels of $c G A S$ and MAVS were associated with an increased risk of CPL (Tables S5 and S6). As shown in Figure 3A, the individuals with hrHPV infection and $c$ GAS methylation levels above $31.87 \%$ might increase the 7.70-fold risk of CPL compared with the others, who had $c G A S$ methylation levels lower than or equal to $31.87 \%$ and were hrHPV-negative $\left(\mathrm{OR}^{\mathrm{a}}=7.70,95 \% \mathrm{CI}=2.84-20.88, \mathrm{P}^{\mathrm{a}}<\right.$ 0.001). Moreover, compared with the individuals who had MAVS methylation levels less than or equal to $21.16 \%$ without hrHPV infection, the risk of CPL was 4.33-fold higher in those who were hrHPV-positive and had MAVS methylation levels above $21.16 \%$ $\left(\mathrm{OR}^{\mathrm{a}}=4.33,95 \% \mathrm{CI}=1.78-10.52, P^{\mathrm{a}}=0.001\right)($ Figure 3B$)$.

The MDR analysis was used to test the interaction among gene promoter methylation levels, hrHPV infection and some other variables; however, multifactor interaction models were not found in CPL or CC (Table S7).

\section{DISCUSSION}

This is the first study that describes the roles of DNA methylation in the promoter regions of the important innate immune pathways of cGAS, MAVS, and TRAF3 in CPL and CC. The methylation levels in the promoter regions of $c G A S, M A V S$, and TRAF3 were significantly increased in the CPL group compared with the healthy control group, while there were no statistically significant differences between the control and CC groups, suggesting that aberrant DNA methylation in the gene promoter regions of $c G A S, M A V S$, and TRAF3 are the early events in the progression of cervical neoplasm.

The carcinogenesis of cervix exhibits the dynamics of DNA methylation changes (Widschwendter et al., 2007; Zhuang et al., 2012; Xia L, 2016), which remains largely unexplored. DNA
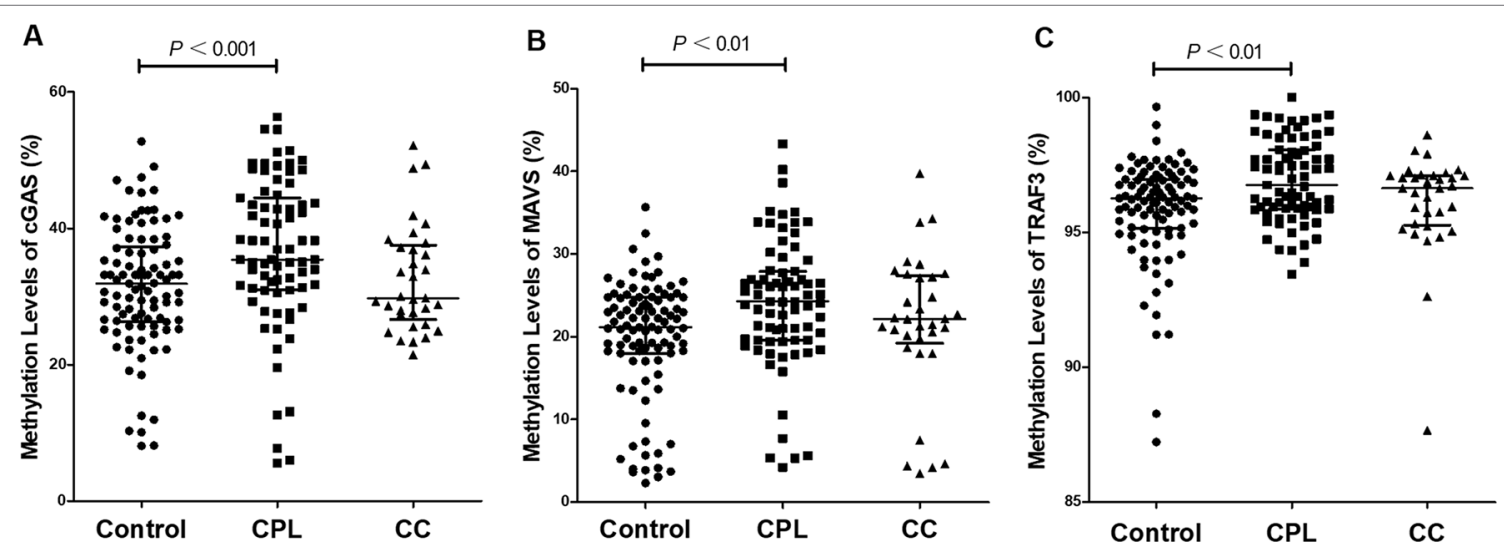

FIGURE 2 | Comparison of gene promoter methylation levels. (A) Cyclic GMP-AMP synthase (cGAS), (B) mitochondrial antiviral-signaling (MAVS), (C) tumor necrosis factor receptor-associated factor 3 (TRAF3). The reference is control group, and differences between pairs were assessed using Mann-Whitney $U$ test. Scatter dot plot represents the methylation level and the line in plot present the median with interquartile range. 
TABLE 3 | Association analysis of the promoter methylation level of cyclic GMP-AMP synthase (cGAS)/mitochondrial antiviral-signaling (MAVS)/tumor necrosis factor receptor-associated factor 3 (TRAF3) gene with the risk on cervical precancerous lesion (CPL), and cervical cancer (CC).

\begin{tabular}{|c|c|c|c|c|c|c|}
\hline \multirow[t]{2}{*}{ Group } & \multicolumn{2}{|c|}{ Methylation level } & \multirow[t]{2}{*}{ OR $(95 \% \mathrm{Cl})$} & \multirow[t]{2}{*}{$P$} & \multirow[t]{2}{*}{$\mathrm{OR}^{\mathrm{a}}(95 \% \mathrm{Cl})$} & \multirow[t]{2}{*}{$P a$} \\
\hline & Low & High & & & & \\
\hline \multicolumn{7}{|l|}{ cGAS } \\
\hline Control & 49 (50.52) & $48(49.48)$ & 1.00 & & 1.00 & \\
\hline CPL & $23(30.67)$ & 52 (69.33) & $2.31(1.23-4.34)$ & 0.009 & $2.49(1.31-4.75)$ & 0.006 \\
\hline $\mathrm{CC}$ & $18(54.55)$ & $15(45.45)$ & $0.85(0.39-1.88)$ & 0.689 & $0.91(0.38-2.19)$ & 0.832 \\
\hline \multicolumn{7}{|l|}{ MAVS } \\
\hline Control & $49(50.52)$ & $48(49.48)$ & & & & ・ \\
\hline CPL & 26 (34.67) & 49 (65.33) & $1.92(1.04-3.58)$ & 0.039 & 1.97 (1.06-3.69) & 0.033 \\
\hline $\mathrm{CC}$ & 13 (39.39) & $20(60.61)$ & $1.57(0.70-3.51)$ & 0.271 & $1.48(0.62-3.52)$ & 0.380 \\
\hline \multicolumn{7}{|l|}{ TRAF3 } \\
\hline Control & 49 (50.52) & $48(49.48)$ & & & & \\
\hline CPL & 31 (41.33) & $44(58.67)$ & $1.45(0.79-2.66)$ & 0.232 & $1.45(0.79-2.66)$ & 0.237 \\
\hline $\mathrm{CC}$ & 13 (39.39) & $20(60.61)$ & $1.57(0.70-3.51)$ & 0.271 & $2.21(0.90-5.43)$ & 0.083 \\
\hline
\end{tabular}

Low, cGAS $\leqq 31.87 \%$, MAVS $\leqq 21.16 \%$, TRAF3 $\leqq 96.26 \%$; High, cGAS > 31.87\%, MAVS > 21.16\%, TRAF3 > 96.26\%. Statistically significant $p$ values (p < 0.05) in bold font was based on logistic regression. ${ }^{a}$ Adjusted odds ratio (OR) on age and number of pregnancies.
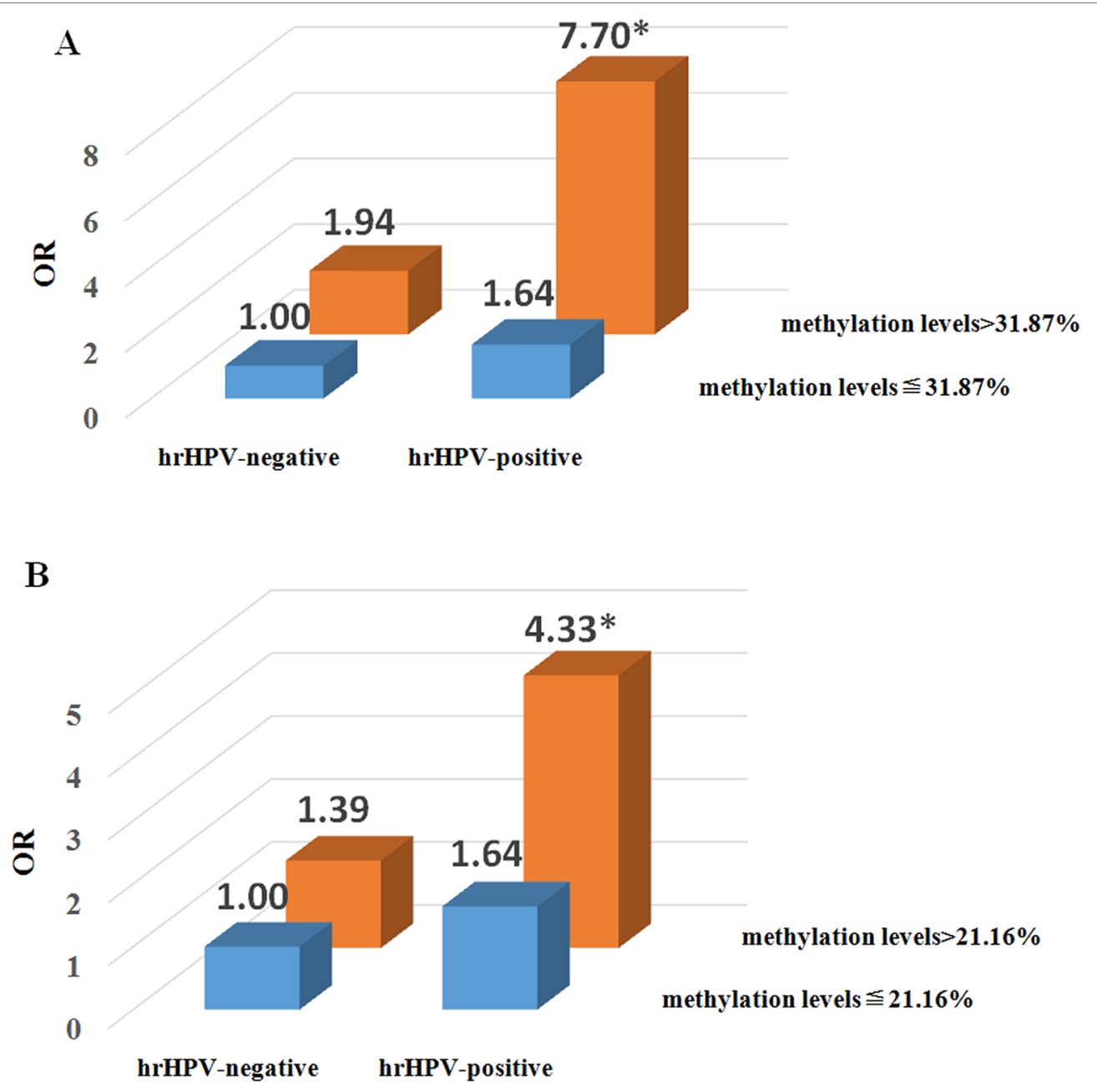

FIGURE 3 | Risk analysis of the two-factor interaction of high-risk human papillomavirus (hrHPV) and promoter methylation levels in the cervical precancerous lesion (CPL). (A) The interaction between hrHPV and methylation level of cyclic GMP-AMP synthase (CGAS). The reference group is the combination of hrHPVnegative (including IrHPV-positive and HPV negative individuals) and $c G A S$ methylation levels $\leqq 31.87 \%$. ${ }^{*} P 0.001$, adjusted odds ratio (OR) on age and number of pregnancies. (B) The interaction between hrHPV and methylation level of mitochondrial antiviral-signaling (MAVS). The reference group is the combination of hrHPVnegative (including IrHPV-positive and HPV negative individuals) and MAVS methylation levels $\leqq 21.16 \% .{ }^{*} P=0.001$, adjusted OR on age and number of pregnancies. 
methylation alterations across the numerous genes are seen even in early carcinogenesis. The cervical cancer model showed that gain of abnormal methylation at some genes in stem cells can be detected up to 3 years in advance of the appearance of precancerous cells, while this process of methylation status was changing during cancer progression (Zhuang et al., 2012). The methylation status might be affected by different factors from the stages of SIL to invasive cancer because the progression may result from the accumulation of genetic and epigenetic alterations with various environmental risks over a long period of time (Armstrong and Doll, 1975; Sadikovic et al., 2008; Szalmás and Kónya, 2009). Increasing evidence has shown that epigenetic changes as a result of aberrant hypermethylation of CpG islands in promoters or histone modifications are essential to carcinogenesis and metastasis (Saavedra et al., 2012). Our study systematically analyzed the promoter methylation status of $c G A S, M A V S$, and TRAF3 genes, which can be identified as new promising methylation markers for the detection of cervical precancerous disease in the early stages.

The patient age and number of pregnancies were significantly higher in the CC group than in the control group, and no significant differences were observed between the CPL and control groups, which was consistent with the results from a study by Castle et al. that showed multiple pregnancies had little or no impact on the development of CPL (Castle et al., 2010). The association between the number of pregnancies and CC risk was also identified in Muñoz's study (Munoz et al., 2002). Some studies revealed that older age was a risk factor for CPL and CC diseases (Netsanet et al., 2016; Teame et al., 2018). The women over 40 years old were at greater risk for invasive CC than those less than 40 years old, and this result could account for the longer period for potential exposure to the HPV virus and the time required for precancerous lesions to develop into invasive cancer (Netsanet et al., 2016). In our study, age was significantly higher only in the CC group than in the control group; this result was similar to that in the Gessesse et al. study, which documented the absence of statistically significant age in CPL (Gessesse et al., 2015).

We found that the elevated methylation levels in $c G A S$ and MAVS promoter regions were risk factors for cervical precancerous disease after adjusting for age and number of pregnancies. The $c G A S / S T I N G$ and RIG-I/MAVS pathways were closely related to HPV infection and to CPL and CC (Lau et al., 2015, Xiao et al., 2016, Hajek et al., 2017), but the mechanisms are still unclear. In our study, the promoter methylation levels of $c G A S$ and MAVS were elevated, which may affect the function of innate immunity of the cGAS/STING and RIG-I/MAVS pathways in the development of CPL. The innate immune responses are acute and temporary in the early stage, and it takes many years or even decades to advance from precancer to invasive cancer in the cervix during persistent HPV infection in women (Winer et al., 2005). Thus, the methylation levels of $c G A S$ and MAVS might gradually become stable during the long progression of CC, with little or no impact on this stage. Abnormal methylation levels of many genes, such as tumor suppressor genes and DNA repair genes, are closely related to CPL and CC diseases (Dong et al., 2001; Feng et al.,
2005; Henken et al., 2007; Jeong et al., 2010; Missaoui et al., 2011b; Li et al., 2015a), and several studies demonstrated that methylation analysis is a potential diagnostic tool for cervical precancerous or cancer screening that may potentially be used alone or in conjunction with cytology and/or HPV(Feng et al., 2005, Missaoui et al., 2011b). Moreover, a study by Bierkens indicated that methylation analysis was capable of accurately detecting HSIL that was missed by cytology, which potentially resulted from the absence of intact indicator cells or cytological sampling errors (Bierkens et al., 2013). Therefore, the aberrant methylation levels of the $c G A S$ and MAVS gene promoters may be an early event in carcinogenesis and could act as potential markers for screening CPL at early stages.

Markedly, we found two synergistic interactions in CPL, which were hrHPV infection with $c G A S$ methylation levels and hrHPV infection with MAVS methylation levels. DNA methyltransferases (DNMTs) are mammalian enzymes responsible for maintaining CpG methylation, and DNMT1 can be activated by both E6 and E7 of hrHPV (Verlaat et al., 2018). E6 can upregulate DNMT1 via p53, and E7 can directly bind to and activate DNMT1 (Burgers et al., 2007; Au Yeung et al., 2010). Conversely, silencing of E6 and E7 could reduce DNA methylation levels and restore the transformed phenotype in CC cells (Rampias et al., 2009; Li et al., 2015b; Verlaat et al., 2018). We hypothesize that DNMT1 is activated by hrHPV that methylation levels in the $c G A S$ and MAVS promoter regions are elevated, and that there are synergistic interactions between the increased $c G A S$ and MAVS methylation levels and hrHPV. Thus, women with hrHPV infection and higher promoter methylation levels in the $c G A S$ and $M A V S$ promoter region might have a higher probability of developing CPL than the others who have lower methylation levels and are hrHPV-negative. In the MDR analysis, we did not identify any gene-gene or gene-environment interaction in CPL or CC, but we cannot rule out the possibility of multiple interactions that have no roles in these diseases. This result may be due to the few environmental factors included in our analysis, so we should collect more demographic data in future research studies.

There are some limitations in our study. First, the sample size was not large enough, among which the number of cases in the CC group was small; this limitation may give rise to the increase in statistical error about probability of category II, leading to test power decrease and being prone to falsenegative results. Therefore, more samples need to be collected for subsequent studies to validate our research. Additionally, the case-control study design may affect the accuracy of the methylation analysis results because of the detection delay times; therefore, we should carry a prospective observational study to dynamically monitor methylation levels at different time points and stages in future studies.

In conclusion, the methylation levels in the promoter regions of $c G A S, M A V S$, and TRAF3 are higher in the CPL group than in the $\mathrm{CC}$ and control group, indicating that hypermethylation in the innate immunity pathway may be an early event in the progression of CIN. The methylation levels in the promoter regions of $c G A S$ and $M A V S$ are related to CPL; moreover, the respective interactions of hrHPV infection with $c G A S$ or MAVS methylation levels may have roles in CPL. Our study provides a 
new epidemiological clue about the role of biomarker screening and the clinical prevention of CPL and CC.

\section{ETHICS STATEMENT}

All patients involved in the study were required to read and sign the informed consent forms for both clinical epidemiological investigation and gene methylation testing prior to enrolment. Each participant was needed to be collected the peripheral venous blood and cervical epithelial exfoliated cells. The study was carried out in accordance with the recommendations of the Helsinki Declaration and the study protocol was approved by the ethics committee of The First Affiliated Hospital of Jinan University.

\section{AUTHOR CONTRIBUTIONS}

CJ and GY contributed to study conception and whole design. $\mathrm{SH}, \mathrm{RL}$, and XH wrote the present paper. SZ, LW, ZW, XZ, JW, YL, $\mathrm{DL}, \mathrm{YW}, \mathrm{SD}, \mathrm{XC}, \mathrm{KZ}, \mathrm{XD}, \mathrm{ZZ}, \mathrm{YH}, \mathrm{XY}, \mathrm{CZ}$, and BZ performed

\section{REFERENCES}

Andersson, T., Alfredsson, L., Kallberg, H., Zdravkovic, S., and Ahlbom, A. (2005). Calculating measures of biological interaction. Eur. J. Epidemiol. 20, 575-579. doi: 10.1007/s10654-005-7835-x

Armstrong, B., and Doll, R. (1975). Environmental factors and cancer incidence and mortality in different countries, with special reference to dietary practices', International. J. Cancer 15, 617. doi: 10.1002/ijc.2910150411

Assmann, S. F., Hosmer, D. W., Lemeshow, S., and Mundt, K. A. (1996). Confidence intervals for measures of interaction. Epidemiology 7, 286-290. doi: 10.1097/00001648-199605000-00012

Au Yeung, C. L., Tsang, W. P., Tsang, T. Y., Co, N. N., Yau, P. L., and Kwok, T. T. (2010). HPV-16 E6 upregulation of DNMT1 through repression of tumor suppressor p53. Oncol. Rep. 24, 1599-1604. doi: 10.3892/or_00001023

Bierkens, M., Hesselink, A. T., Meijer, C. J., Heideman, D. A., Wisman, G. B., Van Der Zee, A. G., et al. (2013). CADM1 and MAL promoter methylation levels in hrHPV-positive cervical scrapes increase proportional to degree and duration of underlying cervical disease. Int. J. Cancer 133, 1293-1299. doi: 10.1002/ ijc. 28138

Bouvard, V., Baan, R., Straif, K., Grosse, Y., Secretan, B., Ghissassi, F. E., et al. (2009). A review of human carcinogens-Part B: biological agents. Lancet Oncol. 10, 321-322. doi: 10.1016/S1470-2045(09)70096-8

Burgers, W. A., Blanchon, L., Pradhan, S., De, L. Y., Kouzarides, T., and Fuks, F. (2007). Viral oncoproteins target the DNA methyltransferases. Oncogene 26, 1650-1655. doi: 10.1038/sj.onc. 1209950

Castle, P. E., Walker, J. M., and Wheeler, C. M. (2010). Hormonal contraceptive use, pregnancy and parity, and the risk of cervical intraepithelial neoplasia 3 among oncogenic HPV DNA-positive women with equivocal or mildly abnormal cytology. Int. J. Cancer 117, 1007-1012. doi: 10.1002/ijc.21279

Chen, W., Zheng, R., Baade, P. D., Zhang, S., Zeng, H., Bray, F., et al. (2016). Cancer statistics in China, 2015. CA A Cancer J. Clinicians 66, 115. doi: 10.3322/ caac. 21338

Chiu, Y. H., Macmillan, J. B., and Chen, Z. J. (2009). RNA Polymerase III Detects Cytosolic DNA and Induces Type-I Interferons Through the RIG-I Pathway. Cell 138, 576. doi: 10.1016/j.cell.2009.06.015

Dong, S. M., Kim, H. S., Rha, S. H., and Sidransky, D. (2001). Promoter hypermethylation of multiple genes in carcinoma of the uterine cervix. Clin. Cancer Res. 7, 1982-1986.

Esteller, M., Hamilton, S. R., Burger, P. C., Baylin, S. B., and Herman, J. G. (1999a). Inactivation of the DNA repair gene O6-methylguanine-DNA the experiments and carried out data collection. All authors approved the final version to be published.

\section{FUNDING}

This work was supported in part by the Major Research Plan of the National Natural Science Foundation of China (91543132), National Natural Science Foundation of China (grant no: 81541070, 30901249, and 81101267), the Guangdong Natural Science Foundation (grant no: 2018A030313601, 10151063201000036, S2011010002526, and 2016A030313089), Guangdong Province Medical Research Foundation (grant no: A2014374, A2015310), and Project from Jinan university (grant no: 21612426, 21615426, JNUPHPM2016001, and JNUPHPM2016002).

\section{SUPPLEMENTARY MATERIAL}

The Supplementary Material for this article can be found online at: https://www.frontiersin.org/articles/10.3389/fgene.2019.01123/ full\#supplementary-material

methyltransferase by promoter hypermethylation is a common event in primary human neoplasia. Cancer Res. 59, 793.

Esteller, M., Sanchez-Cespedes, M., Rosell, R., Sidransky, D., Baylin, SB., and Herman, JG. (1999b). Detection of aberrant promoter hypermethylation of tumor suppressor genes in serum DNA from non-small cell lung cancer patients. Cancer Res. 59, 67-70.

Esteller, M., Sparks, A., Toyota, M., Sanchezcespedes, M., Capella, G., Peinado, M. A., et al. (2000). Analysis of adenomatous polyposis coli promoter hypermethylation in human cancer. Cancer Res. 60, 4366-4371.

Feng, Q., Balasubramanian, A., Hawes, S. E., Toure, P., Sow, P. S., Dem, A., et al. (2005). 'Detection of hypermethylated genes in women with and without cervical neoplasia. J. Natl. Cancer Inst 97, 1548-1549. doi: 10.1093/jnci/dji318

Fitzgerald, K. A., Mcwhirter, S. M., Faia, K. L., Rowe, D. C., Latz, E., Golenbock, D. T., et al. (2003). IKK|[epsi]| and TBK1 are essential components of the IRF3 signaling pathway. Nat. Immunol. 4, 491-496. doi: 10.1038/ni921

Gessesse, Z., Tadesse, Z., Alemayehu, M., Hiruye, A., Getachew, Y., Derbew, M., et al. (2015). Determinant factors of visual inspection with acetic acid (via) positive lesions among hiv positive women in mekelle hospital, northern ethiopia: a case control study. Ethiopian Med. J. Suppl 2, 17.

Guo, W., Zhang, L. W., Guo, Y. L., Yang, Z. B., Kuang, G., and Dong, Z. M. (2009). Methylation status of HLTF gene promoter in the tissues and plasma of gastric cardiac adenocarcinoma and precancerous lesions: a comparative study. Tumor 29, 374-378.

Hajek, M., Sewell, A., Kaech, S., Burtness, B., Yarbrough, W. G., and Issaeva, N. (2017). TRAF3/CYLD mutations identify a distinct subset of human papillomavirus-associated head and neck squamous cell carcinoma. Cancer 123, 1778-1790. doi: 10.1002/cncr.30570

Henken, F. E., Wilting, S. M., Overmeer, R. M., Van Rietschoten, J. G., Nygren, A. O., Errami, A., et al. (2007). Sequential gene promoter methylation during HPV-induced cervical carcinogenesis. Br. J. Cancer 97, 1457-1464. doi: 10.1038/sj.bjc.6604055

Hiscott, J. (2007). Triggering the Innate Antiviral Response through IRF-3 Activation. J. Biol. Chem. 282, 15325-15329. doi: 10.1074/jbc.R700002200

Hornung, V., Ellegast, J., Kim, S., Brzózka, K., Jung, A., Kato, H., et al. (2006). 5-Triphosphate RNA is the ligand for RIG-I. Science 314, 994-997. doi: 10.1126/science. 1132505

Jeong, D. H., Youm, M. Y., Kim, Y. N., Lee, K. B., Sung, M. S., Yoon, H. K., et al. (2010). Promoter methylation of p16, DAPK, CDH1, and TIMP-3 genes in cervical cancer: correlation with clinicopathologic characteristics. Int. J. Gynecological Cancer 16, 1234-1240. doi: 10.1136/ijgc-00009577-200605000-00043 
Karim, R., Tummers, B., Meyers, C., Biryukov, J. L., Alam, S., Backendorf, C., et al. (2013). Human papillomavirus (HPV) upregulates the cellular deubiquitinase UCHL1 to suppress the keratinocyte's innate immune response. PloS Pathog 9, e1003384. doi: 10.1371/journal.ppat.1003384

Kawai, T., Takahashi, K. S., Coban, C., Kumar, H., Kato, H., Ishii, K. J., et al. (2005). IPS-1, an adaptor triggering RIG-I- and Mda5-mediated type I interferon induction. Nat. Immunol. 6, 981-988. doi: 10.1038/ni1243

Lau, L., Gray, E. E., Brunette, R. L., and Stetson, D. B. (2015). DNA tumor virus oncogenes antagonize the cGAS-STING DNA-sensing pathway. Science 350, 568-571. doi: 10.1126/science.aab3291

Lendvai, G., Johannes, F., Grimm, C., Eijsink, J. J. H., Wardenaar, R., Volders, H. H., et al. (2012). Genome-wide methylation profiling identifies hypermethylated biomarkers in high-grade cervical intraepithelial neoplasia. Epigenetics 7, 1268. doi: 10.4161/epi.22301

Li, J. Y., Huang, T., Zhang, C., Jiang, D. J., Hong, Q. X., Ji, H. H., et al. (2015a). Association between RASSF1A Promoter Hypermethylation and Oncogenic HPV Infection Status in Invasive Cervical Cancer: a Meta-analysis. Asian Pacific J. Cancer Prev. Apjcp 16, 5749-5754. doi: 10.7314/APJCP.2015.16.14.5749

Li, J., Kang, L. N., and Qiao, Y. L. (2011). Review of the cervical cancer disease burden in mainland China. Asian Pac J. Cancer Prev. 12, 1149-1153.

Li, L. C., and Dahiya, R. (2002). MethPrimer: designing primers for methylation PCRs. Bioinformatics 18, 1427-1431. doi: 10.1093/bioinformatics/18.11.1427

Li, L., Xu, C., Long, J., Shen, D., Zhou, W., Zhou, Q., et al. (2015b). E6 and E7 gene silencing results in decreased methylation of tumor suppressor genes and induces phenotype transformation of human cervical carcinoma cell lines. Oncotarget 6, 23930-23943. doi: 10.18632/oncotarget.4525

Matthys, V. S., Cimica, V., Dalrymple, N. A., Glennon, N. B., Bianco, C., and Mackow, E. R. (2014). Hantavirus GnT elements mediate TRAF3 binding and inhibit RIG-I/TBK1-directed beta interferon transcription by blocking IRF3 phosphorylation. J. Virol. 88, 2246-2259. doi: 10.1128/JVI.02647-13

Maucort-Boulch, D., Franceschi, S., and Plummer, M. (2008). International correlation between human papillomavirus prevalence and cervical cancer incidence. Cancer Epidemiol. Biomarkers Prev.: Publ. Am. Assoc. Cancer Res. cosponsored by Am. Soc. Preventive Oncol. 17, 717. doi: 10.1158/1055-9965. EPI-07-2691

Merlo, A., Herman, J. G., Mao, L., Lee, D. J., Gabrielson, E., Burger, P. C., et al. (1995). '5' CpG island methylation is associated with transcriptional silencing of the tumour suppressor p16/CDKN2/MTS1 in human cancers. Nat. Med. 1, 686-692. doi: 10.1038/nm0795-686

Missaoui, N., Hmissa, S., Trabelsi, A., Traore, C., Mokni, M., Dante, R., et al. (2011b). Promoter hypermethylation of CDH13, DAPK1 and TWIST1 genes in precancerous and cancerous lesions of the uterine cervix. Pathol. Res. Pract. 207, 37-42. doi: 10.1016/j.prp.2010.11.001

Missaoui, N., Hmissa, S., Trabelsi, A., Traoré, C., Mokni, M., Dante, R., et al. (2011a). Promoter hypermethylation of CDH13, DAPK1 and TWIST1 genes in precancerous and cancerous lesions of the uterine cervix. Pathol. Res. Pract. 207, 37. doi: 10.1016/j.prp.2010.11.001

Mj, K., Pe, C., At, L., W., M., Dr, S., et al. (2005). The elevated 10-year risk of cervical precancer and cancer in women with human papillomavirus (HPV) type 16 or 18 and the possible utility of type-specific HPV testing in clinical practice. J. Natl. Cancer Institute 97, 1072. doi: 10.1093/jnci/dji187

Munoz, N., Franceschi, S., Bosetti, C., Moreno, V., Herrero, R., Smith, J. S., et al. (2002). Role of parity and human papillomavirus in cervical cancer: the IARC multicentric case-control study. Lancet 359, 1093-1101. doi: 10.1016/ S0140-6736(02)08151-5

Netsanet, S., Graciela, S. D., Konjit, K., Brooks, M. I., Teklu, W., Yewondwossen, T., et al. (2016). The Single-Visit Approach as a Cervical Cancer Prevention Strategy Among Women With HIV in Ethiopia: Successes and Lessons Learned. Glob Health Sci. Pract. 4, 87-98. doi: 10.9745/GHSP-D-15-00325

Nojima, D., Nakajima, K., Li, L. C., Franks, J., Ribeirofilho, L., Ishii, N., et al. (2001). CpG methylation of promoter region inactivates E-cadherin gene in renal cell carcinoma. Mol. Carcinog 32, 19-27. doi: 10.1002/mc.1060

Oganesyan, G., Saha, S. K., Guo, B., He, J. Q., Shahangian, A., Zarnegar, B., et al. (2006). Critical role of TRAF3 in the Toll-like receptor-dependent and -independent antiviral response. Nature 439, 208-211. doi: 10.1038/ nature 04374
Peto, J., Gilham, C., Deacon, J., Taylor, C., Evans, C., Binns, W., et al. (2004a). Cervical HPV infection and neoplasia in a large population-based prospective study: the Manchester cohort. Br. J. Cancer 91, 942-953. doi: 10.1038/ sj.bjc. 6602049

Peto, J., Gilham, C., Fletcher, O., and Matthews, F. E. (2004b). The cervical cancer epidemic that screening has prevented in the UK. Lancet 364, 249-256. doi: 10.1016/S0140-6736(04)16674-9

Pichlmair, A., Schulz, O., Tan, C. P., Näslund, T. I., Liljeström, P., Weber, F., et al. (2006). RIG-I-mediated antiviral responses to single-stranded RNA bearing 5'-phosphates. Science 314, 997-1001. doi: 10.1126/science.1132998

Rampias, T., Sasaki, C., Weinberger, P., and Psyrri, A. (2009). E6 and E7 Gene Silencing and Transformed Phenotype of Human Papillomavirus 16-Positive Oropharyngeal Cancer Cells. J. Natl. Cancer Inst 101, 412-423. doi: 10.1093/ jnci/djp017

Ribeiro-Filho, L. A., Franks, J., Sasaki, M., Shiina, H., Li, L. C., Nojima, D., et al. (2002). CpG hypermethylation of promoter region and inactivation of E-cadherin gene in human bladder cancer. Mol. Carcinogenesis 34, 187. doi: $10.1002 / \mathrm{mc} .10064$

Saavedra, K. P., Brebi, P. M., and Roa, J. C. S. (2012). Epigenetic alterations in preneoplastic and neoplastic lesions of the cervix. Clin. Epigenet. 4, 13. doi: 10.1186/1868-7083-4-13

Sadikovic, B., Alromaih, K., Squire, J. A., and Zielenska, M. (2008). Cause and Consequences of Genetic and Epigenetic Alterations in Human Cancer. Curr. Genomics 9(6):394-408. doi: 10.2174/138920208785699580

Shi, J. F., Canfell, K., Lew, J. B., and Qiao, Y. L. (2012). The burden of cervical cancer in China: Synthesis of the evidence. Int. J. Cancer 130, 641. doi: 10.1002/ ijc. 26042

Skrondal, A. (2003). Interaction as departure from additivity in case-control studies: a cautionary note. Am. J. Epidemiol. 158, 251-258. doi: 10.1093/aje/ kwg113

Steenbergen, R. D., Snijders, P. J., Heideman, D. A., and Meijer, C. J. (2014). Clinical implications of (epi)genetic changes in $\mathrm{HPV}$-induced cervical precancerous lesions. Nat. Rev. Cancer 14, 395-405. doi: 10.1038/nrc3728

Sun, L., Wu, J., Du, F., Chen, X., and Chen, Z. J. (2013). Cyclic GMP-AMP Synthase Is a Cytosolic DNA Sensor That Activates the Type I Interferon Pathway. Science 339, 786-791. doi: 10.1126/science. 1232458

Szalmás, A., and Kónya, J. (2009). Epigenetic alterations in cervical carcinogenesis. Semin. Cancer Biol. 19, 144-152. doi: 10.1016/j.semcancer.2009.02.011

Teame, H., Addissie, A., Ayele, W., Hirpa, S., Gebremariam, A., Gebreheat, G., et al. (2018). Factors associated with cervical precancerous lesions among women screened for cervical cancer in addis ababa, ethiopia: a case control study. PloS One 13, e0191506. doi: 10.1371/journal.pone.0191506

Verlaat, W., Van Leeuwen, R. W., Novianti, P. W., Schuuring, E., Meijer, C., Van Der Zee, A. G. J., et al. (2018). Host-cell DNA methylation patterns during highrisk HPV-induced carcinogenesis reveal a heterogeneous nature of cervical pre-cancer. Epigenetics. 13(7), 769-778. doi: 10.1080/15592294.2018.1507197

Wentzensen, N., Sherman, M., and Wang, S. (2009). Utility of methylation markers in cervical cancer early detection: appraisal of the state-of-the-science. Gynecologic Oncol. 112, 293-299. doi: 10.1016/j.ygyno.2008.10.012

Widschwendter, M., Fiegl, H., Egle, D., Mueller-Holzner, E., Spizzo, G., Marth, C., et al. (2007). Epigenetic stem cell signature in cancer. Nat. Genet. 39, 157-158. doi: $10.1038 /$ ng 1941

Winer, R. L., Kiviat, N. B., Hughes, J. P., Adam, D. E., Lee, S. K., Kuypers, J. M., et al. (2005). Development and Duration of Human Papillomavirus Lesions, after Initial Infection. J. Infect. Dis. 191, 731-738. doi: 10.1086/427557

Wu, J., Sun, L., Chen, X., Du, F., Shi, H., Chen, C., et al. (2013). Cyclic GMP-AMP is an endogenous second messenger in innate immune signaling by cytosolic DNA. Science 339, 826-830. doi: 10.1126/science.1229963

Xia L, L. T., Tan, Qiufen, Yuling, D., Xiaolin, P., Lijuan, P., Yan, Qi, et al. (2016). CpG island methylation of the CADM1 gene correlates with cervical carcinogenesis in the Uighur and Han populations of Xinjiang, China. Int. J. Clin. Exp. Pathol. 9 (7), 6977-6987.

Xiao, D., Huang, W., Ou, M., Guo, C., Ye, X., Liu, Y., et al. (2016). 'Interaction between susceptibility loci in cGAS-STING pathway, MHC gene and HPV infection on the risk of cervical precancerous lesions in Chinese population. Oncotarget 7, 84228. doi: 10.18632/oncotarget.12399 
Zhao, F. H., Lewkowitz, A. K., Hu, S. Y., Chen, F., Li, L. Y., Zhang, Q. M., et al. (2012). Prevalence of human papillomavirus and cervical intraepithelial neoplasia in China: A pooled analysis of 17 population-based studies. Int. J. Cancer 131, 2929-2938. doi: 10.1002/ijc.27571

Zhuang, J., Jones, A., Lee, S. H., Ng, E., Fiegl, H., Zikan, M., et al. (2012). The dynamics and prognostic potential of DNA methylation changes at stem cell gene loci in women's cancer. PloS Genet. 8, e1002517. doi: 10.1371/journal.pgen.1002517

Zou, X. P., Zhang, B., Zhang, X. Q., Chen, M., Cao, J., and Liu, W. J. (2009). 'Promoter hypermethylation of multiple genes in early gastric adenocarcinoma and precancerous lesions. Hum. Pathol. 40, 1534-1542. doi: 10.1016/j. humpath.2009.01.029
Conflict of Interest: The authors declare that the research was conducted in the absence of any commercial or financial relationships that could be construed as a potential conflict of interest.

Copyright $\odot 2019$ Huang, Li, Huang, Zheng, Wang, Wen, Zou, Wu, Liu, Liu, Wang, Dong, Chen, Zhu, Du, Zhou, Han, Ye, Zeng, Zhang, Yang and Jing. This is an openaccess article distributed under the terms of the Creative Commons Attribution License (CC BY). The use, distribution or reproduction in other forums is permitted, provided the original author(s) and the copyright owner(s) are credited and that the original publication in this journal is cited, in accordance with accepted academic practice. No use, distribution or reproduction is permitted which does not comply with these terms. 